\title{
Correction to: Oseledets Splitting and Invariant Manifolds on Fields of Banach Spaces
}

\author{
Mazyar Ghani Varzaneh ${ }^{1,2} \cdot$ S. Riedel ${ }^{1}$
}

Published online: 9 April 2021

○ Springer Science+Business Media, LLC, part of Springer Nature 2021

\section{Correction to: Journal of Dynamics and Differential Equations https://doi.org/10.1007/s10884-021-09969-1}

The original version of this article unfortunately contained a typo in co-author name.

The author name is "Mazyar Ghani Varzaneh" instead it was published incorrectly as "M. Mazyar Ghani Varzaneh".

The original article has been corrected.

The original article can be found online at https://doi.org/10.1007/s10884-021-09969-1.

\section{S. Riedel}

riedel@math.tu-berlin.de

Mazyar Ghani Varzaneh

mazyarghani69@gmail.com

1 Institut Für Mathematik, Technische Universität Berlin, Berlin, Germany

2 Department of Mathematical Sciences, Sharif University of Technology, Tehran, Iran 